\title{
2. Prozesse der Subjektivation: Die Unterwerfung, das Psychische und der Widerstand
}

In der theoretischen Bewegung von der Heteronormativität zum Homonationalismus geht es immer auch um Prozesse der Subjektivierung. Es geht um Geschlechternormen, die intelligible Subjekte produzieren, um neoliberale Regierungstechniken, die Subjekte dazu bringen, sich selbst zu regieren, oder um die Produktion kolonisierter Subjekte. Identität kann als ein Prozess der Subjektwerdung verstanden werden, der das Subjekt zu totalisieren versucht. Diesem Streben nach Schließung im Prozess der Subjektwerdung versucht das queere politische Subjekt zu entkommen.

Im Folgenden soll entsprechend vertiefend dargestellt werden, wie Prozesse der Subjektivation (queer-)theoretisch verstanden werden können, welche Rolle dabei Identität spielt und wie Widerstand - im Modus des nicht derart Regiert-Werdens - gedacht werden kann. Hierzu soll zunächst mit Louis Althusser die freiwillige Unterwerfung im Prozess der Subjektwerdung näher in den Blick genommen werden. Die Ideologietheorie von Althusser verweist bereits darauf, dass das identitätspolitische Subjekt der Befreiung selbst immer schon unterworfen ist. Anschließend werden, Bezug nehmend auf Judith Butler, die mit den Prozessen der Subjektwerdung einhergehenden Formierungen des Psychischen näher betrachtet. Die Formierungen des Psychischen verweisen auf einen »listigeren Weg ", in dem die Macht operiert, ${ }^{1}$ und ermöglichen eine Kritik an Identität als Name der Befreiung auf der Ebene der psychischen Subjektivation. ${ }^{2}$ Abschließend werden in Anlehnung an die queer-theoretischen Denker*innen Judith Butler und José Esteban Muñoz Möglichkeiten des Widerstandes gegen die unterwerfende, zum Teil totalisierende, Subjekte konstituierende Macht ausgelotet.

1 Judith Butler: Psyche der Macht. Das Subjekt der Unterwerfung, Frankfurt a.M.: Suhrkamp Verlag 2015, S. 25.

2 Hanna Meißner: Jenseits des autonomen Subjekts. Zur gesellschaftlichen Konstitution von Handlungsfähigkeit im Anschluss an Butler, Foucault und Marx, Bielefeld: transcript Verlag 2010, S. 65. 


\subsection{Die freiwillige Unterwerfung}

Louis Althusser versucht in einem seiner berühmtesten Aufsätze »Ideologie und ideologische Staatsapparate (ISA)«, der erstmals 1970 auf Französisch erschienen ist, aufzuzeigen, wie Menschen zu Subjekten werden. Zentral ist der Begriff der Ideologie, den er in Abgrenzung zu einem Verständnis vom »falschen Bewusstsein « und von der Vorstellung einer notwendigen Übereinstimmung von ideologischer Position und Position im Produktionsprozess einsetzt. ${ }^{3}$ Das wesentlichste beziehungsweise der am meisten rezipierte Punkt der Althusser'schen Ideologietheorie ist, dass die Ideologie Subjekte hervorbringt, indem sie die Individuen anruft. Als Beispiel für ein solches Anrufungsszenario führt Althusser den Polizisten an, der auf der Straße »Hey, Sie da« ruft. In dem Moment, in dem die angerufene Person sich umdreht - eine »einfache physische Wendung um 180 Grad« vollzieht -, erkennt sie an, dass sie gemeint ist, und wird als Subjekt konstituiert. ${ }^{4}$

Ausgangspunkt von Althussers Ideologietheorie ist aber zunächst die Klärung der Frage nach der »Reproduktion der Produktionsbedingungen« über die zu erlernenden (körperlichen) Fähigkeiten hinaus. ${ }^{5}$ Dieser Teil wird in der Rezeption häufig vergessen. ${ }^{6}$ Althusser konstatiert, dass es zur Reproduktion der Arbeitskraft nicht ausreiche, dass die Arbeitskraft die für die Produktion nötigen Qualifikationen erlernt. Es ist ebenso notwendig, so seine These, dass sie sich den Regeln der herrschenden Ordnung unterwirft: »Mit anderen Worten: die Schule (aber auch andere Institutionen des Staates wie die Kirche oder andere Apparate wie die Armee) lehren >Fähigkeiten<, aber in Formen, die die Unterwerfung unter die herrschende Ideologie oder die Beherrschung ihrer >Praxis sichern ${ }^{7}{ }^{7}$ Butler hebt in diesem Zusammenhang hervor, dass Althusser bereits in dieser Formulierung die Opposition Unterwerfung/Beherrschung aufhebt. Sich der Ideologie zu unterwerfen, heißt, eine Praxis zu beherrschen. ${ }^{8}$ Die Orte, an denen die für die Reproduktion der Produktionsbedingungen notwendigen Unterwerfungen stattfinden, sind die ideologischen Staatsapparate, die Althusser von den repressiven Staatsapparaten unterscheidet: »der repressive Staatsapparat > funktioniert auf der Grundlage der Gewalt‘, während die ideologischen Staatsapparate - >aufder Grundlage der Ideologie funktionieren. ${ }^{9}$ Es sind also die ideologischen Staatsapparate, die ebenjene Unterwer-

Stuart Hall: Ideologie, Identität, Repräsentation. Ausgewählte Schriften 4, Hamburg: Argument Verlag 2016, S. 42.

4 Louis Althusser: Ideologie und ideologische Staatsapparate. Aufsätze zur marxistischen Theorie, Hamburg: VSA Verlag 1977, S. $142 f$.

5 Ebd., S. $108 \mathrm{f}$.

6 S. Hall: Ideologie, Identität, Repräsentation, S. 58.

7 L. Althusser: Ideologie und ideologische Staatsapparate, S. 112.

8 J. Butler: Psyche der Macht, S. 109.

9 L. Althusser: Ideologie und ideologische Staatsapparate, S. 121. Beide Staatsapparate funktionieren jeweils auch über Ideologie und Cewalt. Nur ist im repressiven Staatsapparat Gewalt der dominante Mechanismus, während im ideologischen Staatsapparat die Ideologie zentral ist. Althusser zählt eine ganze Reihe von Institutionen auf, die er als ideologische Staatsapparate versteht: die Religion, die Schule, die Familie, der juristische Staatsapparat, der politische Staatsapparat, die Gewerkschaften, die Medien und der kulturelle Staatsapparat. Vgl. L. Althusser: Ideologie und ideologische Staatsapparate, S. $119 f$. 
fungen und Beherrschung einer Praxis lehren, die notwendig für die Reproduktion der Produktionsverhältnisse sind.

So wichtig dieser erste Teil aus Althussers Essay auch ist - beschreibt er doch, wie die Konstitution von Subjekten die Reproduktion der Produktionsbedingungen ermöglicht -, so sehr sind einige Ausführungen aus diesem Teil auch in die Kritik geraten. Foucault beispielsweise kritisiert, ohne explizit Althussers Namen zu nennen, ein Machtverständnis, nachdem die Mechanismen der Macht auf Instrumente reduziert werden, die allein der Reproduktion der Produktionsverhältnisse dienen. Dementgegen will Foucault die über die Reproduktion der Produktionsverhältnisse hinausgehenden Machtverhältnisse in den Blick nehmen. ${ }^{10}$ Er kritisiert aber auch die Rolle, die Althusser den Staatsapparaten zuschreibt, und setzt dieser sein eigenes Machtverständnis entgegen: »Der Staatsapparat scheint mir eher eine konzentrierte Form beziehungsweise stützende Struktur eines Machtsystems zu sein, das darüber hinausreicht und sehr viel tiefer geht. ${ }^{11}$ Auch die Unterscheidung zwischen repressivem und ideologischem Staatsapparat greift aus Foucault'scher Sicht zu kurz. In ihr sei eine Interpretation von Ideologie als falsch, Illusion oder vielleicht sogar falsches Bewusstsein angelegt. Der ideologische Staatsapparat kann, so Warren Montag, im Unterschied zum repressiven, gewaltvollen Staatsapparat nur die Indoktrination von Ideen sein, die Menschen $\mathrm{zu}$ Subjekten machen und sich in bestimmten Handlungen ausdrücken: »By opposing it to the violence of the Repressive Apparatuses, however, he appears to endorse a political dualism of force and consent ${ }^{12} .{ }^{12}$ Auch Foucault scheint sich von diesem vermeintlichen Dualismus in Althussers Denken abzugrenzen, wenn er sich gegen ein Verständnis von Macht wendet, die entweder als Gewalt oder als Ideologie funktioniert:

»Doch die Macht ist nicht in der Alternative gefangen, sich entweder schlicht und einfach mit Gewalt durchzusetzen oder sich zu verstecken, für ihre Distanz zu sorgen, indem sie den geschwätzigen Diskurs der Ideologie hält. Tatsächlich ist jede Machtausübung zugleich auch ein Ort der Ausbildung nicht einer Ideologie, sondern des Wissens; und umgekehrt erlaubt und gewährleistet jedes allgemeingültige Wissen die Ausübung von Macht. Anders gesagt, gilt es nicht, eine Opposition aufzumachen, zwischen dem was getan wird, und dem, was gesagt wird, dem Schweigen der Cewalt und der Geschwätzigkeit der Ideologie [...]. $^{13}$

Althusser versucht also im ersten Teil von »ISA« die Rolle der Ideologie als Ort, an dem die Reproduktion der Produktionsverhältnisse gesichert wird, einzuordnen. Dabei entwickelt er eine Staatstheorie, die unter anderem dafür kritisiert wird, dass sie erstens

10 Michel Foucault: Die Strafgesellschaft. Vorlesung am Collège de France 1972-1973, Berlin: Suhrkamp Verlag 2015, S. 237.

11 Ebd., S. 312.

12 Warren Montag: ») The Soul is the Prison of the Body<. Althusser and Foucault, 1970-1975«, in: Yale French Studies (1995), S. 53-77, hier S. 61.

13 M. Foucault: Die Strafgesellschaft, S. 317. An dieser Stelle setzten die Herausgeber des Bandes (Francois Ewald, Alessandro Fontana und Bernard E. Harcourt) eine Fußnote, in der sie darauf verweisen, dass sich die hier zitierte Passage gegen Althussers Artikel »Ideologie und ideologische Staatsapparate« richtet. M. Foucault: Die Strafgesellschaft, S. 329. 
zu kurz greife und zweitens eine Opposition zwischen Gewalt und Ideologie - Materiellem und Ideellem - aufmache. Isolde Charim hingegen kritisiert eine Opposition zwischen Althusser'scher Ideologietheorie und Foucault'scher Machttheorie. Sie versteht erstere ebenfalls als Machttheorie. ${ }^{14}$ Althusser selbst scheint zudem im zweiten Teil seines Essays selbst den ihm vorgeworfenen Dualismus aufzuheben, wenn er von der $»$ materiellen Existenz der Ideologie« spricht. ${ }^{15}$

\section{Die materielle und die imaginäre Form der Ideologie}

Althusser formuliert im zweiten Teil seines Essays zunächst zwei positive Thesen über die Ideologie. Erstens hat die Ideologie eine »imaginäre Form ${ }^{16}$ und zweitens eine »materielle Existenz «. ${ }^{17}$ Im Gegensatz zu einer Vorstellung von Ideologie als Idee oder Weltanschauung, die Handlungen begründet, hat Ideologie bei Althusser eine spezifische Materialität. Sie hat insofern eine materielle Existenz, als sie immer in einem »Apparat und dessen Praxis « existiert. ${ }^{18}$ Um das Verhältnis von Ideen, Praxen, Apparaten und Handlungen in der Ideologie zu fassen, bedient sich Althusser des bekannten Zitats von Blaise Pascal, das er aus dem Kopf heraus zitiert: »Knie nieder, bewege die Lippen zum Gebet, und Du wirst glauben. «" Althusser will damit keine bloße Umkehrung des ideellen und materiellen Verhältnisses begründen, sondern eine »eigenartige Umbildung ${ }^{20}$ Es ist, wie Isolde Charim ausführt, nicht so, dass das Materielle (»knie nieder«) das Ideelle (»du wirst glauben«) als Zentrum ablöst. Vielmehr bezeichnet Althusser eine eigene Materialität, eine bestimmte Art der Verbindung verschiedener (ideeller wie materieller) Elemente. ${ }^{21}$ Das Knien ist nicht die Ursache des Glaubens, es hat aber den Effekt, dass geglaubt wird. ${ }^{22}$ Es handelt sich nicht um ein Kausalverhältnis, sondern um eine Zirkularität, in der sich die Unterscheidung zwischen Materiellem und Ideellem auflöst. ${ }^{23}$ Es sind, Isolde Charim zufolge, die spezifischen Verbindungen von Handlungen, Apparaten, Praktiken, Ritualen und Ideen, die die Materialität von Ideologien ausmachen und Subjekte produzieren. ${ }^{24}$ Die These von der materiellen Existenz der Ideologie vermag also einen einfachen Dualismus zwischen Ideellem und Materiellem, der scheinbar in Althussers Unterscheidung zwischen repressivem Staatsapparat und ideologischem Staatsapparat angelegt ist, zu unterlaufen.

Die Ideologie hat aber nicht nur eine materielle Existenz, sondern - und das ist die erste positive These in Bezug auf die Ideologie - auch eine »imaginäre Form«: »Die

Isolde Charim: Der Althusser-Effekt. Entwurf einer Ideologietheorie, Wien: Passagen Verlag 2002, S. 17.

15 J. Butler: Psyche der Macht, S. 114.

16 L. Althusser: Ideologie und ideologische Staatsapparate, S. 133.

17 W. Montag: >The Soul is the Prison of the Body«, S. 62.

18 L. Althusser: Ideologie und ideologische Staatsapparate, S. 137.

19 Ebd., S. 138.

20 I. Charim: Der Althusser-Effekt, S. 79.

21 Ebd., S. 75.

22 Ebd., S. 81.

23 Ebd., S. 82.

24 Ebd., S. 12. 
Ideologie repräsentiert das imaginäre Verhältnis der Individuen zu ihren realen Existenzbedingungen. ${ }^{25}$ Das heißt, die Menschen imaginieren sich nicht ihre realen Existenzbedingungen, sondern vor allem ihr Verhältnis zu diesen Bedingungen. Imaginär ist das Verhältnis, Isolde Charim zufolge, wenn ein Übergang von einer offenen zu einer geschlossenen Struktur stattfindet. Das heißt, wenn das Individuum freiwillig den zugewiesenen Platz einnimmt, also den richtigen Gebrauch der Objekte annimmt. Die Struktur ist dann insofern geschlossen, als sich der Gebrauch auf ein Zentrum - einen "Steppunkt « - bezieht. ${ }^{26}$ Die Subjektkonstitution kann demnach als ein auf Schließung abzielender Prozess verstanden werden, der um ein Zentrum (das SUBJEKT) herum angeordnet ist. ${ }^{27}$

Ein wesentlicher Aspekt der Subjektkonstitution ist zudem, dass die Individuen sich freiwillig auf einen Platz verweisen lassen: »das Individuum wird als (freies) Subjekt angerufen, damit es sich freiwillig den Anordnungen des SUBJEKTS unterwirft, damit es also (freiwillig) seine Unterwerfung akzeptiert und folglich sganz von allein die Gesten und Handlungen seiner Unterwerfung >vollzieht.${ }^{28}$ Das Subjekt verkennt dabei den Zwang seiner Freiwilligkeit. Die wahre Unterwerfung des Subjekts ist bei Althusser unbewusst. ${ }^{29}$ Sie wird durch den Bezug auf die Freiwilligkeit verneint. ${ }^{30}$ In ihr verkennen die Subjekte, dass sie nur für die Unterwerfung und damit letztendlich für die Reproduktion der Produktionsverhältnisse existieren. ${ }^{31}$

\section{Das spiegelhafte Identifizieren}

Damit Individuen sich freiwillig unterwerfen, müssen sie, wie Isolde Charim ausführt, den Platz, auf den sie angerufen werden, als ihren Platz erkennen. Hier spielt die spiegelhafte Struktur der Anrufung eine zentrale Rolle. In den mehrfachen Bedeutungen des Wortes reconnaissance (wiederkennen, anerkennen) findet sich das Schlüsselprinzip des spiegelhaften Identifizierens wieder. ${ }^{32}$ Zunächst muss das Individuum sich im Spiegelbild (wieder-)erkennen, um freiwillig den Platz einzunehmen, an den es gerufen wird. ${ }^{33}$ Dieser Prozess des Wiedererkennens geht jedoch mit einer doppelten Verkennung einher. Indem das Individuum sich im Spiegelbild erkennt, verkennt es, dass eigentlich es selbst die Instanz ist, die den Bezug zum Bild herstellt: »Sich als Subjekt der Aussage zu erkennen heißt, sich als Subjekt der Aussage zu verkennen. ${ }^{34}$ Gleichzeitig verkennt es auch, dass die Anrufung ein performativer Prozess ist, der das Subjekt erst herstellt. Im Prozess der Wiederholung setzt das Individuum ein vorgängiges Subjekt

L. Althusser: Ideologie und ideologische Staatsapparate, S. 133.

I. Charim: Der Althusser-Effekt, S. $147 f$.

Ebd., S. 157. Althusser spricht in diesem Zusammenhang vom großen SUB]EKT als den Punkt, der das Zentrum bildet. Das große SUBJEKT garantiert den Subjekten, dass sie es sind, es ist aber auch abhängig von der Anerkennung durch die Subjekte.

L. Althusser: Ideologie und ideologische Staatsapparate, S. 148.

Hier setzt Butler zufolge die Formierung des Psychischen ein.

I. Charim: Der Althusser-Effekt, S. 160 f.

L. Althusser: Ideologie und ideologische Staatsapparate, S. 149.

I. Charim: Der Althusser-Effekt, S. 150.

Ebd., S. 152.

Ebd., S. 153. 
voraus, das wiederzuerkennen ist: "Es entsteht der Schein, die Identität würde ihrer Identifizierung vorausgehen. Das Subjekt erscheint als Voraussetzung und nicht als Folge der Anrufung. « ${ }^{35}$ In der zweiten Bedeutung des Wortes reconnaissance spielt die wechselseitige Anerkennung zwischen dem großen Subjekt, das im Zentrum der Anrufung steht, und dem angerufenen Individuum eine entscheidende Rolle. Das große Subjekt - beispielsweise Gott oder die Pflicht - gibt den angerufenen Subjekten die Garantie, dass sie es sind, indem es sie anerkennt. Umgekehrt muss auch das angerufene Subjekt die geschlossene Struktur des Platzes, auf den es verwiesen wird, anerkennen. Denn auch diese wird erst im Akt der Subjektwerdung hervorgebracht - ist dieser also nicht vorgängig. Es bedarf also dieses zirkulären und doppelt performativen Prozesses des Identifizierens, der sich um den Begriff der reconnaissance anordnet, damit mehrere Individuen um ein Zentrum herum aneinandergebunden werden können.

Althusser entwirft in »Ideologie und Ideologische Staatsapparate « folglich den Versuch einer Theorie, die beschreibt, wie Machtverhältnisse Subjekte konstituieren, indem sie auf einen bestimmten Platz verwiesen werden. Mehrere Menschen werden durch eine geschlossene Struktur - die gemeinsame Identität, in die sie angerufen werden - aneinandergebunden und determiniert. ${ }^{36}$ Indem die Ideologie Subjekte gleichzeitig produziert und unterwirft, das handelnde Subjekt also gleichzeitig in der und für die Unterwerfung existiert, ist im Althusser'schen Ideologiebegriff bereits die Kritik am Subjekt als Ausgangspunkt der Befreiung angelegt: »Damit schockiert Althusser alle Aufklärungsutopien, die sich an das Subjekt als den Träger der Befreiung wenden. « ${ }^{37}$ Der Modus der Freiwilligkeit, in dem die Unterwerfung unbewusst bleiben muss, verweist zudem bereits auf die Formierungen des Psychischen, die mit Prozessen der Subjektwerdung einhergehen.

Stuart Hall vermisst bei Althusser allerdings die theoretische Auseinandersetzung mit der Frage nach der Selbst-Konstitution der Subjekte: Althusser wisse nicht zu erklären, warum die Subjekte sich (nicht derart) umdrehen, um dem Ruf zu folgen. Es bleibt offen »[...] wie die Einzelnen diese Positionen formen, stilisieren, herstellen und sverkörpern`, warum sie dies nie ein für alle Mal vollständig umsetzen, warum manche dies gar nicht tun oder warum manche in einem fortwährenden, agonistischen Prozess mit Normen und Regeln - mit denen sie sich selbst konfrontieren und sich selbst regulieren - kämpfen, sich diesen Normen und Regeln anpassen, sie verhandeln oder ihnen widerstehen. $\aleph^{38}$ Auch Foucault, der eine Machttheorie entwickelte, in der ebenfalls die gleichzeitige Produktion und Unterwerfung des Subjekts zentral ist, vermochte Hall zufolge zwar »eine diskursive Phänomenologie des Subjektes und eine Genealogie der Technologien des Selbst « zu entwerfen; indem er aber für immer die Tür zur Psychoanalyse und damit zum Unbewussten verschlossen habe, vernachlässige er zwangsläufig zentrale Aspekte der Selbst-Konstitution. ${ }^{39}$ Judith Butler ist eine der Theoretiker*innen, die versucht hat, diese Lücke zu schließen, indem sie Bezug nehmend auf Althus-

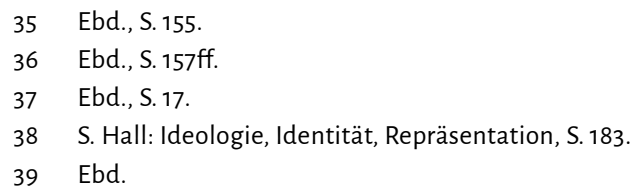


ser und Foucault die mit der Subjektwerdung einhergehenden psychischen Dimensionen in den Blick nimmt. In dem 1997 auf Englisch und 2002 auf Deutsch erschienenen Werk »Psyche der Macht« versucht sie aufzuzeigen, wie die Formierung des Psychischen mit Prozessen der Subjektkonstitution einhergeht. Diese theoretischen Auseinandersetzungen von Judith Butler sind nicht unumstritten. Ein prominenter Vertreter einer solchen Kritik ist beispielsweise Didier Eribon. Er ist der Meinung, dass Foucault eine Verknüpfung seiner subjekttheoretischen Überlegungen mit psychoanalytischen Theorien nie gewollt hätte. Zudem kritisiert er am Beispiel von Lacan-Zitaten die psychoanalytischen Theorien zugrunde liegende Heteronormativität, die nicht mit queertheoretischen Überlegungen vereinbar sei. ${ }^{40}$ Andere betonen wiederum die Bedeutung des Versuches von Judith Butler, eine Brücke zwischen Psychischem und Diskursivem zu schlagen, die es möglich macht, ein komplexeres Bild einer Macht zu zeichnen, die Subjekte konstituiert. ${ }^{41}$

\subsection{Die Formierung des Psychischen}

Subjektivation bedeutet für Judith Butler zum einen Unterordnung unter die Macht, zum anderen gehen damit "gewollte Wirkungen des Subjekts einher ${ }^{42}$ Diese "gewollten Wirkungen« lassen sich wiederum unterscheiden in den Aspekt der freiwilligen Unterwerfung und den Aspekt der widerständigen Handlungsfähigkeit. ${ }^{43}$ Wie ist es möglich, sich als ein durch eine vorgängige Macht konstituiertes Subjekt gegen eben jene Macht zu wenden, die Voraussetzung der eigenen Existenz ist? Und warum unterwerfen Subjekte sich freiwillig der herrschenden Ordnung? In »Psyche der Macht« (der Genitiv bezeichnet »sowohl das `Zugehören` zur Macht wie die `Ausübung der Macht«) möchte Judith Butler die »für immer geschlossene Tür« in der Foucault'schen Theorie öffnen, indem sie einer Erklärung der Subjektivation auch eine Wendung des psychischen Lebens hinzufügt:

»Foucaults Schweigen zum Thema Psyche ist bekannt, aber eine Erklärung der Subjektivation muß, so scheint es, in die Wendung des psychischen Lebens hineinführen. Cenauer muß sie in der merkwürdigen Wendung des Subjekts gegen sich selbst gesucht werden, wie wir sie in Selbstvorwurf, Cewissen und Melancholie vor uns haben, die mit Prozessen sozialer Reglementierung einhergehen. ${ }^{44}$

Das Gewissen und die Melancholie sind Formen der Wendung des Subjekts gegen sich selbst, die Butler als Formierungen des Psychischen in Prozessen der Subjektivation beschreibt. Diese Ausführungen von Butler ermöglichen nicht nur ein vertiefendes Verständnis der Prozesse der Subjektwerdung auf der Seite des Subjekts, sondern auch, das Funktionieren der Macht auf einem »listigeren Weg« als dem des »offenen Zwang[s]«

Vgl. Didier Eribon: Der Psychoanalyse entkommen, Wien: Turia + Kant Verlag 2017. 
$\mathrm{zu}$ fassen. ${ }^{45}$ Was hat es nun aber mit den Wendungen des Subjekts gegen sich selbst auf sich? Wie formieren sich Melancholie und Gewissen im Verhältnis zu sozialen Reglementierungen und auf welche Art und Weise stehen sie in Beziehung zueinander?

\section{"Das Gewissen macht Subjekte aus uns allen"}

Auch Judith Butler wendet sich der Althusser'schen Ideologietheorie zu, um auf die von Stuart Hall benannte theoretische Leerstelle im Anrufungsszenario zu verweisen. Althusser vermag, Butler zufolge, nicht zu erklären, welche Voraussetzungen gegeben sein müssen, damit das Subjekt sich umwendet und sagt »Hier bin ich«:

»[W]o und wann bewirkt der Ruf des Namens diese Umwendung, die vorwegnehmende Bewegung in Richtung Identität? Wie und weshalb wendet sich das Subjekt in Erwartung der Verleihung einer Identität durch die Selbstzuschreibung von Schuld um? Welche Art von Beziehung bindet diese beiden bereits, so daß das Subjekt weiß, wie es sich umzuwenden hat, weiß, daß es etwas dabei zu gewinnen hat? ${ }^{46}$

In »Ideologie und ideologische Staatsapparate«schreibt Althusser zu dieser Frage lediglich, dass es »jedenfalls ein merkwürdiges Phänomen« sei, »das nicht allein durch ein >Schuldgefühk erklärt werden kann ${ }^{47}{ }^{47}$ Auf der Suche nach einer Antwort ergänzt Butler die Interpellationstheorie um eine Theorie des Gewissens: »Das Gewissen ist grundlegend für die Hervorbringung und Reglementierung des Bürgersubjekts, denn das Gewissen wendet das Individuum um und macht es der subjektivierenden Maßregelung zugänglich. ${ }^{48}$ Wie aber bildet sich dieses Gewissen, das eine Beziehung zum Gesetz noch vor der Anrufung beschreibt? Bezug nehmend auf Freud und Nietzsche entsteht das Gewissen, Butler zufolge, durch die Verinnerlichung gesellschaftlicher Verbote, die einen Zirkel der Reflexion anstoßen, der wiederum das ursprüngliche Begehren, sich aufzulösen, in Richtung Subjektivation neu ausrichtet. ${ }^{49}$ Das Gewissen ordnet also das Begehren in eine Richtung an in der die eigene Subjektwerdung zum Ziel wird. Judith Butler beschreibt zwei Formen des Verbotes/Verlustes, die sich über das Gewissen in die Subjektbildung einschreiben und die Grenze der Selbstreflexion markieren: zum einen die Verleugnung der eigenen Abhängigkeit, zum anderen der - der Subjektkonstitution vorausgehende - Verlust in der melancholischen Identifizierung.

Die erste Form des Verbotes geht mit dem durch das Gewissen neu ausgerichteten Ziel der Subjektwerdung einher. Um dieses Ziel zu erreichen, muss der Preis der freiwilligen Unterwerfung in Kauf genommen werden, um gesellschaftlich zu existieren: »Denn das Gewissen, das den Passanten zwingt, sich auf den Ruf des Polizisten umzuwenden [...], scheint getrieben von einer Liebe zum Gesetz. ${ }^{50}$ Das Subjekt begehrt, von der Autorität gesehen zu werden, dabei wird der Preis des Gesehenwerdens - nämlich die Unterordnung - verkannt. Das verinnerlichte Verbot nimmt in diesem 
Modus die Form der Verleugnung der eigenen Unterwerfung und Abhängigkeit an. In der umwendenden Bewegung Richtung Identität wird verleugnet, dass kein Subjekt entstehen kann, ohne die leidenschaftliche Verhaftung an jene, von denen es abhängig ist. ${ }^{51}$ Die Autonomie und Einheit, die Identität verspricht, kann nur über eine Verleugnung der eigenen Abhängigkeit - der grundlegenden Bindungen an die Anderen - hergestellt werden. Insofern, als im Prozess der Subjektwerdung zwangsläufig die eigene Abhängigkeit verdrängt wird, kann die Subjektwerdung nur mit einer Formierung des Unbewussten einhergehen: »Wenn der Autonomieeffekt durch Unterordnung bedingt ist und diese Gründungsunterordnung oder Abhängigkeit rigoros verdrängt wird, dann entsteht das Subjekt zusammen mit dem Unbewussten. ${ }^{52}$

Die psychische Subjektivation verweist darauf, wie Modi der Macht einerseits die Möglichkeit der Reflexion erzeugen, während sie andererseits Formen der Gesellschaftlichkeit begrenzen, indem diese nicht hinterfragt werden können. Die Hinterfragung des Gesetzes ist immer auch insofern beschränkt, als sie auch eine Kritik am eigenen Sein darstellt. ${ }^{53}$ Das Gewissen, das uns zu Subjekten macht, die ihre eigene Abhängigkeit von den anderen verleugnen, setzt Möglichkeiten der Reflexion in Gang, die an ihre Grenzen stößt, wenn es um die Einheit und Autonomie des Ichs geht. Wir wollen die Modi der Macht nur so weit hinterfragen, wie es eine Verleugnung unserer konstitutiven Sozialität zulässt: »Die Anrufung ist vom Erfolg nicht durch eine strukturell fortbestehende Form des Verbots (oder Ausschlusses) >barriert،, sondern durch ihre Unfähigkeit zur Bestimmung des konstitutiven Feld des Menschlichen. ${ }^{54}$ Die »Ordnung des Verbotes« kann aber auch »außerhalb des Zirkels der Selbstreflexion« liegen, wenn sie das Subjekt über ein von Anfang an verworfenes Begehren konstituiert. Es wird über einen vorgängigen Verlust konstituiert, der ebenfalls die Grenze der Reflexion markiert. Dieses Verbot nimmt die Form der melancholischen Identifizierung an. ${ }^{55}$

\section{Melancholische Identifizierung - unbetrauerbare Verluste}

Die die Interpellationstheorie ergänzende Theorie des Gewissens beschreibt, wie eine vom Gewissen getriebene narzisstische Verhaftetheit in der eigenen Weiterexistenz der umwendenden Bewegung in Richtung Identität vorausgeht. Die Formierung des Psychischen, die in den Subjektivationsprozess eingeschlossen ist, ermöglicht die freiwillige Unterwerfung, indem die eigene Abhängigkeit ins Unbewusste verdrängt wird. Aber auch der Selbstvorwurf und die Melancholie sind Mechanismen der psychischen Wendung im Prozess der Subjektwerdung. Bezug nehmend auf Freuds Ausführungen zur melancholischen Identifizierung in das »Ich und das Es« beschreibt Judith Butler zunächst, wie Geschlechtszugehörigkeit über den Ausschluss des homosexuellen Begehrens als melancholische Identifizierung verstanden werden kann. Dabei macht Butler ihr eigenes Schreiben explizit als »kulturelles Engagement in der psychoanalytischen Theorie, das weder zum Gebiet der Psychologie noch der Psychoanalyse gehört«, zu der

$\begin{array}{ll}51 & \text { Ebd., S. } 12 . \\ 52 & \text { Ebd. } \\ 53 & \text { Ebd., S. } 103 . \\ 54 & \text { Ebd., S. } 121 . \\ 55 & \text { Ebd., S. 27. }\end{array}$


sie aber »eine intellektuelle Beziehung [...] herstellen will«, kenntlich. ${ }^{56}$ Sie möchte ihre Unternehmungen als einen Versuch verstanden wissen, produktive Übereinstimmungen zwischen Freuds Vorstellungen von unbetrauerten Verlusten und einer Kultur, in der homosexuelle Verluste schwer zu betrauern sind, aufzuzeigen.

Grundsätzlich wird in der melancholischen Identifizierung ein von Anfang an verlorenes oder verbotenes Objekt, das nicht betrauerbar ist, im Inneren bewahrt, indem es im Prozess der Identifizierung einverleibt wird. ${ }^{57}$ Das verlorene Objekt kann nur aufbewahrt werden, indem das Selbst sich mit dem Objekt identifiziert. Auch vergeschlechtlichte Zugehörigkeit wird wesentlich in Prozessen der melancholischen Identifizierung hervorgebracht. ${ }^{58}$ So kommt die geschlechtliche Identifizierung zumindest zum Teil durch die Abweisung homosexuellen Begehrens zustande. Das Mädchen darf die Mutter nicht begehren und verinnerlicht stattdessen das Verbot, indem es sich mit dem verlorenen Objekt identifiziert. Es wird zu der Frau, die es nicht lieben darf: »In diesem Sinne lassen sich sowohl Männlichkeit wie Weiblichkeit als durch Identifizierungen geformt auffassen, die zum Teil aus verleugneter Trauer bestehen. «59 Das homosexuelle Begehren muss also geopfert werden und um dieses Opfer kann nicht getrauert werden, weil es von Anfang an verworfen ist. Judith Butler spricht von einer doppelten Verleugnung, die das heterosexuelle Subjekt begründet: »nie geliebt und nie verloren zu haben « ${ }^{60}$ Sara Ahmed beschreibt eine Szene aus dem Film »If These Walls Could Talk«, in der dieser doppelte Verlust zu Tage tritt. Nach dem Tod ihrer Lebenspartnerin Edith ist Abby mit deren Familie konfrontiert, die ihre lesbische Trauer nicht wahrnimmt, weil sie die lesbische Beziehung nicht sieht: "Es muss sehr traurig für dich sein, so eine gute Freundin zu verlieren.< Worauf Edith auf unangemessene Weise entgegnet:

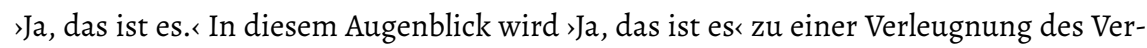
lustes, zu einem Weg, den wahren Verlust geheim zu halten, zu einem Weg, um das geheim zu halten, was als Geheimnis verloren war. ${ }^{61}$ Indem Abby bestätigt, dass es traurig sei, eine gute Freundin verloren $\mathrm{zu}$ haben, verleugnet sie, geliebt und verloren zu haben.

Bezug nehmend auf die Aids-Krise fragt Butler, ob in einer ganzen Kultur, in der homosexuelles Begehren unbetrauerbar ist, eine Kultur der Geschlechtermelancholie entstehe. Hier beginnt sie, die aktuellen historisch-politischen Bedingungen, die homosexuelle Verluste im Zuge der Aids-Krise als unbetrauerbar wiederholen und ritualisieren, produktiv mit psychoanalytischen Überlegungen zur melancholischen Identifizierung als spezifischer psychischer Ökonomie in Beziehung zu setzen. Welche Rolle aber spielt die Melancholie in Bezug auf die Reglementierungsmacht? In welchem Verhältnis stehen beide zueinander? Butler selbst schreibt, dass es nicht ganz klar sei, wie die Beziehungen zwischen sozialer und psychischer Welt in Bezug auf die Melancholie zu denken sind. Die Melancholie kann aber erklären, wie diese beiden Berei-

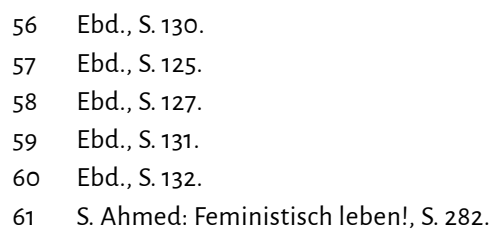


che »in Beziehung zueinander hervorgebracht werden $« .{ }^{62}$ Auch hier spielt das Gewissen eine zentrale Rolle. Es kann mit Freud als eine Instanz und Institution verstanden werden, durch die Melancholie hervorgebracht und erhalten wird. ${ }^{63}$ Gesellschaftliche Ideale werden in einem Prozess der Verinnerlichung zu einem Schuldgefühl, das sich gegen sich selbst wendet und die Struktur des Gewissens hervorbringt. Dabei bleibt in diesen Prozessen unsichtbar, wie die reglementierende Macht ihre Spuren im idealisierten Gewissen weiterträgt. ${ }^{64}$ Unsichtbar bleibt auch, dass in der Melancholie die »topographische Unterscheidung zwischen Ich und Über-Ich« erst hergestellt wird. Sie sind der Melancholie nicht vorgängig. Das, was der Melancholie als Verlust einverleibt ist, ist die soziale Welt selbst: »Das Ich wird ein >Gemeinwesen seiner 'großen Institutionen<, eben weil das psychische Leben im Versuch, die Verluste dieser Welt auszulöschen, eine soziale Welt in sich selbst hinein zurücknimmt. ${ }^{65}$ Die soziale Welt speist das Gewissen insofern, als sie entscheidet, welches Leben betrauerbar ist und welches nicht. ${ }^{66}$ In der Aids-Krise waren es beispielsweise die Leben der Homosexuellen, Drogenabhängigen und People of Color, die als nicht betrauerbar galten.

Das Gewissen reguliert nicht in Form von Verboten oder Normen, sondern von Selbstvorwürfen und Aggression. Selbstvorwürfe und Aggression sind Effekte unerfüllbarer Ideale, mit denen das Ich konfrontiert wird. ${ }^{67}$ Das homosexuelle Begehren, das von Anfang an verloren ist, wird in Form von Selbstvorwurf und Schuld nicht nach außen, sondern gegen sich selbst gewendet. ${ }^{68}$ Indem die unbetrauerbaren Verluste der sozialen Welt im Prozess der Melancholie verinnerlicht werden, entsteht eine Struktur des Gewissens, das Subjekte reguliert, indem die Verbote/Verluste in Form von Aggression gegen es selbst gewendet werden. Melancholie und Macht bringen sich auch insofern wechselseitig hervor, als die Melancholie »die psychischen Bedingungen dafür fest[schreibt], die >Welt « selbst [als] durch ganz bestimmte Arten des Ausschlusses organisiert zu betrachten ${ }^{69}$ Die im Psychischen verworfenen Identifizierungen, die sich nach außen hin nicht zeigen, grenzen in gewisser Hinsicht die Identifizierungen ein, die sich nach außen zeigen: »die heterosexuelle Frau wird zu der Frau, die sie niemals liebte und um die sie niemals trauerte. In diesem Sinn ist also dasjenige, was höchst offenkundig als soziale Geschlechtsidentität darstellerisch realisiert wird, das Zeichen und Symptom einer alles durchdringenden Verleugnung. " $^{70}$

Daran anschließend schärft Butler auf der Ebene der psychischen Subjektivation auch ihre Kritik an Politiken, die Identität zum gemeinsamen Ausgangspunkt politischer Praxis machen. ${ }^{71}$ Wenn Geschlechtszugehörigkeit auf einer konstitutiven Verwer- 
fung gründet, dann entsteht auch die Homosexualität in einem konstitutiven Bezug zur Heterosexualität. Wird eine lesbische oder schwule Identität bejaht, müssen die »heterosexuellen Reste eine solchen Identität genau dadurch bewahrt werden, daß man auf der nahtlosen Kohärenz einer spezifischen schwulen Identität beharrt «. ${ }^{72}$ Dementgegen schlägt Butler vor, die Möglichkeiten des Widerstandes in der Artikulation solcher "Ambivalenzen« und »Verluste«, durch die eine Offenheit und Beweglichkeit hergestellt werden kann, zu suchen. ${ }^{73}$

Während also Althusser beschreibt, wie die Ideologie Subjekte konstituiert, versucht Butler in ihren Ausführungen zur Melancholie und zum Gewissen, die damit einhergehenden Formierungen des Psychischen zu fassen: Zum einen erklärt eine leidenschaftliche Verhaftetheit in der eigenen Existenz, warum Subjekte sich freiwillig unterwerfen, zum anderen wird im Moment der Unterwerfung das Unbewusste erzeugt, weil der Zwang und die leidenschaftliche Bindung an den Anderen verleugnet werden müssen, um sich selbst als autonomes, selbstidentisches Subjekt konstituieren zu können. Im Prozess der melancholischen Identifizierung verinnerlicht das Ich die soziale Welt, um deren Verluste im Inneren zu bewahren. So wird die Welt als eine Welt des Ausschlusses erfahrbar. Das in den Prozessen der melancholischen Identifizierung verinnerlichte soziale Ideal erzeugt eine Struktur des Gewissens, das die Wut über den Verlust gegen das Selbst richtet. Psychisches und Diskursives sind, diesen Überlegungen zufolge, miteinander verwoben und konstituieren sich gegenseitig. Sie haben aber auch ihre jeweils eigene Ökonomie. Identität bedeutet folglich zum einen Unterwerfung unter die auf einen Platz verweisende Macht, zum anderen gehen mit Prozessen der Identifizierung Verinnerlichungen einher, die die Macht hervorbringen, die sie hervorgebracht hat. Das Psychische trägt dazu bei zu verstehen, warum das Subjekt sich freiwillig unterwirft. Es hat dabei eine soziale Existenz zu gewinnen. Wir wissen aber auch, was es dabei zu verlieren hat, und dieser Verlust wendet sich meist in Form von Wut und Selbstvorwürfen gegen das Selbst. Sie kann aber auch nach außen gerichtet und politisiert werden, womit wir beim zweiten Aspekt der "gewollten Wirkungen« der Subjektivation sind: der widerständigen Handlungsfähigkeit. ${ }^{74}$

\subsection{Queerer Widerstand}

Ein auf Althusser, Foucault und Butler Bezug nehmendes Verständnis von Subjektivation geht davon aus, dass das Subjekt der Unterwerfung gleichzeitig als potentiell widerständiges Subjekt produziert wird. Wie kann aber ein Widerstand gegen die Macht aussehen, wenn das widerständige Subjekt von Anfang an unterworfen ist. Wie kann eben jene geschlossene Struktur, die, Althusser zufolge, Individuen zu Subjekten macht und sie einander bindet, indem sie sie determiniert, aufgebrochen oder unterlaufen werden? Wie kann ihr widerstanden werden und welche Rolle spielen dabei die 
Dimensionen des Psychischen? Diesen Fragen widmen sich ausgehend von den vorangegangen theoretischen Überlegungen viele queere Theoriebildungen insofern, als "queer« als ein Ort verstanden wird, der zum Ziel hat, eben jene geschlossene, imaginäre Struktur, die Subjekte unterwirft - aber auch Menschen aneinander bindet - , offenzuhalten. Judith Butler versucht - die soeben beschriebenen Dimensionen des Psychischen berücksichtigend - in ihren älteren Werken, queeren Widerstand, am Beispiel von Drag-Performances und der Politisierung der Wut in der Aids-Krise, als theatralische Art und Weise, den »Verlusten eine Sprache« zu geben, zu fassen. Der queere Theoretiker of Color José Esteban Muñoz nimmt die Möglichkeiten der Verkennung im anrufungstheoretischen Prozess der Identifizierung zum Ausgangspunkt, um mit Blick auf Performance-Künstler*innen of Color queeren Widerstand als disidentification $\mathrm{zu}$ beschreiben. Dabei versteht er disidentification als eine Strategie des Überlebens solcher Leben, deren Verluste als unbetrauerbar gelten. Auch in Judith Butlers jüngsten Überlegungen zur pluralen Performativität spielen unbetrauerbare Verluste eine zentrale Rolle. Ausgehend von einer Politik, die die im Prozess der Subjektwerdung verleugnete Abhängigkeit zum Ausgangspunkt nimmt, soll eine ungleiche Betrauerbarkeit von Leben politisiert werden.

\subsubsection{Theatralisches Zitieren: Den Verlusten eine Sprache geben}

Ähnlich wie bei Althusser und Foucault steht auch bei Judith Butler der Prozess der Subjektwerdung als gleichzeitiger Akt der Unterwerfung und der Produktion des handelnden Subjekts im Zentrum der Überlegungen. Eine wichtige Rolle spielt dabei der Begriff der Performativität. Er beschreibt den körperlichen/sprachlichen Akt, in dem Subjekte hergestellt werden. ${ }^{75}$ An Jacques Derrida anschließend, bringt Butler die Iterabilität als notwendiges Moment der performativen Praxis ins Spiel, das rein technisch gesehen Möglichkeiten des Widerstandes eröffnet. Die Tatsache, dass die Herstellung von Geschlechtsidentitäten ein Prozess ist, der im sprechenden oder körperlichen Akt ständig wiederholt werden muss, ermöglicht ein falsches Zitieren der Norm. ${ }^{76}$ Die Wiederholung ist anfällig für Fehler. ${ }^{77}$ Entsprechend können im Akt der Wiederholung die Konstruktionsprozesse von Identitäten offengelegt werden. ${ }^{78}$ Aber auch performativitätstheoretisch begründete Formen des (queeren) Widerstandes kommen nicht umhin, Identitäten, die auf einen bestimmten Platz verweisen, einzusetzen:

»In diesem Sinne bleibt es unverzichtbar auf die Begriffe >Frauen<, >queer >lesbisch<Anspruch zu erheben, und zwar genau der Form wegen, in der sie sozusagen Anspruch auf uns erheben, bevor wir darum ganz wissen. Es ist nötig, umgekehrt auf solche Begriffe Anspruch zu erheben, um ihre homosexuellenfeindliche Verwendung 
im Recht, in der öffentlichen Politik, auf der Straße und im >Privatleben< zu verhindern. ${ }^{79}$

Es ist notwendig, verletzende, ein- und ausschließende sowie hierarchisierende Begriffe einzusetzen, weil politische Begriffe nicht einfach aus dem Nichts geschöpft werden können. Auch queerer Widerstand muss Anspruch erheben auf Begriffe, die eine Geschichte haben, die niemals ganz von ihnen losgelöst werden kann. Sie können aber für die Zukunft neu imaginiert werden, indem sie ihrer Bedeutungen enthoben und rekontextualisiert werden. ${ }^{80} \mathrm{Um}$ die neu imaginierte Zukunft selbst nicht wieder festzuschreiben, muss »queer ein »Ort kollektiver Auseinandersetzungen« bleiben, »Ausgangspunkt für eine Reihe historischer Überlegungen und Zukunftsvorstellungen«, der immer mehr ist, als er gerade zu beschreiben vermag, und der ständig »neu eingesetzt«, "umgedreht « und »gequeert (durchkreuzt) « wird. ${ }^{81}$ Ein kollektives Wir, das sich um den Begriff »queer « anordnet, ist diesen Überlegungen zufolge keine Gemeinschaft, die sich auf einen Platz verweisen lässt. »Queer« bedeutet nicht zu sagen, genau das sind wir, sondern immer wieder neu zu fragen, wer oder was dieses Wir sein will.

Wie kann ein queerer Widerstand, der die auf Schließung abzielenden Prozesse der Subjektwerdung offenhalten will, aussehen? Als eine Möglichkeit queeren Widerstandes führt Butler die Geste der theatralischen Zitierung ein. Die notwendige Wiederholung, in der Identitäten immer wieder neu eingesetzt werden, ermöglicht es mit Hilfe von theatralischen Gesten, hegemoniale Bedeutungsanordnungen $\mathrm{zu}$ unterlaufen und anders zu imaginieren:

»Diese Art von Zitierung wird in dem Maße als theatralische Zitierung in Erscheinung treten, in dem sie die diskursive Konvention nachahmt und übertreibt, die sie zudem auch umgekehrt. Die übertriebene Geste ist entscheidend für die Bloßstellung des homosexuellenfeindlichen `Gesetzes«, das die Begriffe seiner eigenen verwerflich machenden Strategien nicht mehr kontrollieren kann. ${ }^{82}$

So ist die Tatsache, dass "queer « als ehemaliges Schimpfwort im US-amerikanischen Raum angeeignet und mit einer positiven Bedeutung versehen wurde, ein Versuch, auf theatralische Weise den Begriff einzusetzen, durch den queere Personen über Jahre hinweg schmerzhaft eingesetzt wurden. "Queer « anzueignen, bedeutete, »die spezifische Geschichtlichkeit des Begriffs aufzudecken, zu bejahen und umzugestalten.«" spielte die Wut als eine Dimension des psychischen Prozesses der Subjektwerdung eine zentrale Rolle. Dem »Queer Nation Manifesto« zufolge ist die Bejahung des Begriffs "queer« ein Ausdruck von Wut: »But when a lot of lesbians and gay men wake up in the morning we feel angry and disgusted, not gay. So we've chosen to call ourselves queer. ${ }^{84}$ Judith Butler versteht die in der theatralischen Geste politisierte Wut als ei-

\footnotetext{
79 J. Butler: Körper von Gewicht, S. 314.

80 Ebd., S. $212 f$.

81 Ebd., S. 313.

82 Ebd., S. 319.

83 Ebd., S. 316.

84 Queer Nation: THE QUEER NATION MANIFESTO 1990, https://www.historyisaweapon.com/defcon 1/queernation.html vom 29.05.2020.
} 
nen Effekt der melancholischen Identifizierung in einer Kultur, in der homosexuelle Verluste unbetrauerbar sind. ${ }^{85}$ Den Namen "queer « anzunehmen, hieß, Ja zu sagen zu einer Wut, die sich aufgrund der Geschichte und Erfahrungen der Verletzungen angestaut hatte und die in der Aids-Krise neue Dimensionen entwickelte. Dabei handelt es sich um affektive Formen der Politisierung. Es ist die Wut, die in der Melancholie gegen sich selbst gerichtet wird, die in den Politisierungen der Aids-Krise nach außen gerichtet wurde. Es wurden kollektive Trauerprozesse ins Leben gerufen, um die Betrauerbarkeit der unbetrauerbaren Verluste einzufordern. ${ }^{86}$ Als weitere Beispiele für »übertriebene Gesten«, in denen die Wut über die im Zuge der Aids-Krise erfahrenen Verletzungen und unbetrauerbaren Verluste in Form von theatralischen Zitierungen politisiert wurde, führt Butler »Die-ins«, »Kiss-ins«, »Drag balls«, »Street walking« und "Butch.femme spectacles « an. ${ }^{87}$ So wurde beispielsweise in Form von »Die-ins « das Sterben im Zuge der Aids-Krise öffentlich gemacht, und »Kiss-ins« dienten dazu, auf die Ausschlüsse von Homosexualität aus dem öffentlichen Raum aufmerksam zu machen. Dabei geht es darum, den (körperlichen/sprachlichen) Akt, der ein Sein einsetzt, das unbetrauerbar ist, in der theatralischen Geste offenzulegen, zu rekontextualisieren und ihm eine neue Bedeutung zu geben.

Das wohl bekannteste Beispiel, das Butler für die Politisierung unbetrauerbarer Verluste anführt, ist die Drag-Performance. Performance beschreibt im Gegensatz zur Performativität die »darstellerische Realisierung als begrenzter Akt«. Drag vermag es, das "Wahrheits-Regime des >Sexes« zu kritisieren, indem die Darstellung die melancholische Einverleibung unbetrauerbarer Verluste »allegorisiert «. ${ }^{88}$ Die Darstellung allegorisiert eine heterosexuelle Melancholie, die aus der Unbetrauerbarkeit des Verlustes der gleichgeschlechtlichen Liebe resultiert. Denn - wie Butler in Anlehnung an Freud ausführt - eine übertriebene Männlichkeit ist das Resultat der Einverleibung des homosexuellen Verlustes: keinen Mann lieben zu können. Drag als eine gegengeschlechtliche Darstellung versinnbildlicht diese heterosexuelle Melancholie: »Was drag gleichwohl aufdeckt, ist die >normale< Verfaßtheit der Präsentation sozialer Geschlechtsidentität, in der das dargestellte Geschlecht in vielen Hinsichten durch eine Reihe verleugneter Neigungen oder Identifizierungen konstituiert wird, die einen andersartigen Bereich des >Undurchführbaren begründen. ${ }^{89}$ Drag vermag nicht die Vielfältigkeiten von Geschlechtern zu begründen, sondern auf deren Verluste $\mathrm{zu}$ verweisen und eine andere Zukünftigkeit $\mathrm{zu}$ imaginieren. ${ }^{90}$

Theatralisches Zitieren ist Butler zufolge also eine Möglichkeit, die über Ausschluss und Verwerfungen hergestellten vergeschlechtlichten Identitäten bloßzustellen und so teilzuhaben an den Verfahren, in denen Subjekte über Verwerfungen und Ausschlüsse hergestellt werden. Die auf einen Platz verweisende geschlossene Struktur der Anrufung wird offen und beweglich gehalten, indem den damit einhergehenden Verlusten 
eine Sprache gegeben wird. Diesen Überlegungen zufolge findet Widerstand gegen auf eine geschlossene Struktur abzielende Formen der Subjektwerdung zwar nicht im Psychischen statt, das Affektive (Wut und Trauer) spielt aber eine zentrale Rolle. Wut und Trauer tragen nicht nur dazu bei, soziale Reglementierungen auf einem »listigeren« Weg zu festigen, sie können auch Ausgangspunkt (Wut nach außen richten) und Gegenstand (unbetrauerbare Verluste sozialer Reglementierungen offenlegen) des Widerstandes gegen die Macht sein. Dabei geht es darum, in der theatralischen Geste die notwendige Wiederholung, in der das Subjekt eingesetzt wird, zu nutzen, um eine offenere Zukunft $\mathrm{zu}$ imaginieren.

\subsubsection{Disidentification: Working on and Against Identity}

Ebenfalls inspiriert durch und erarbeitet am Beispiel von Performances, Kunst, aber auch literarischen und medialen Repräsentationen von Queers of Color arbeitet José Esteban Muñoz in dem 1999 erschienenen Buch »Disidentifications. Queers of Color and the Performance of Politics« disidentification als eine Strategie für minorisierte Subjekte heraus. Muñoz verortet sein theoretisches Vorhaben zwischen als konstruktivistisch und essentialistisch geltenden theoretischen Ansprüchen. ${ }^{91}$ Er versteht es als intersektional $^{92}$ und ist beeinflusst von poststrukturalistischen und psychoanalytischen Theoretiker*innen sowie den Cultural Studies. ${ }^{93}$ Im Zentrum seiner Überlegungen steht die Strategie der disidentification, die er, inspiriert durch die Arbeiten queerer Performance-Künstler*innen, als eine mögliche Antwort auf die schmerzhaften Anrufungen, die Queers of Color erfahren, herausarbeitet: »It [disidentification] is a response to state and global power that employ systems of racial, sexual, and national subjugation. These routinized protocols of subjugation are brutal and painful. Disidentification is about managing and negotiating historical trauma and systemic violence. " 4

Während Judith Butler den Blick auf die widerständige Politisierung der mit der Subjektwerdung einhergehenden Verluste legt, bezieht sich disidentification auf die mit dem Prozess der Anrufung einhergehende notwendige Anerkennung und Wiedererkennung. Zentral für seine Überlegungen zur disidentification sind vor allem die Ausführungen von Michel Pêcheux, der Bezug nehmend auf das Althusser'sche AnrufungsSzenario drei Wege der Identifizierung ausgemacht hat. Der erste Weg ist der der klassischen Identifizierung. In Muñoz' Worten ist das der Weg, auf dem die angerufene Person sich vom »Hey, you there! « auf einen heterosexuell strukturierten Platz verweisen lässt. Die zweite Möglichkeit ist die der counteridentification. Das ist der Weg, auf

91 J. E. Muñoz: Disidentifications, S. 6.

92 Ebd., S. 8.

93 Insbesondere »This Bridge Called My Back« von Cherríe Moraga and CloriaE.Anzaldúa (zuerst 1981 veröffentlicht) stellt Muñoz zufolge ein wertvolles Beispiel für eine disidentifikatorische Praxis dar. Entgegen der Kritik von Haritaworn, dass Theoretiker*innen of Color, wie Lorde, Moraga Anzaldúa, die weit davon entfernt waren, den Tod des Subjekts zu begründen, zu »Ceburtshelfer*innen« der queeren Nicht-Identität werden, betont Muñoz die zentrale Bedeutung der beiden Theoretiker*̈innen, sowohl seine eigene Arbeit betreffend als auch generell für queere Genealogien. Ebd., S. 22 
dem das heterosexuelle »Hey, you there« ignoriert wird, was bedeutet, die Anrufung zu verweigern. Doch auch diese Möglichkeit der Verweigerung bleibt in den Strukturen der Anrufungen verhaftet, solange sie eine reine Negation des Heterosexuellen ist. Der dritte Weg ist der queere Weg der disidentification. Disidentification bedeutet, sich weder vollständig mit der Anrufung zu identifizieren, noch sie vollständig abzulehnen, sondern gleichzeitig an ihr, mit ihr und gegen sie zu arbeiten: »disidentification is a strategy that works on and against dominant ideology. ${ }^{95}$ Es bedeutet ähnlich wie in Judith Butlers Ausführungen zur theatralischen Zitierung, nicht ganz auf Bezeichnungen zu verzichten, die einen (schmerzhaft) einsetzten. Disidentification ist eine Strategie, das heteronormative »Hey, you there!« umzuarbeiten, indem die Anrufung bis zu einem bestimmten Grad scheitert: "queers are people who have failed to turn around to the >Hey, you there! interpellating call of heteronormativity. ${ }^{96}$ Dabei spielt die Möglichkeit, im Modus des Wiedererkennens die Anrufung zu verkennen, eine zentrale Rolle. Das Scheitern von Identität - »the failure of identity « - wird zum Ausgangspunkt queerer disidentifikatorischer Praktiken. Am Beispiel von Frantz Fanon, der als wichtiger postkolonialer Theoretiker gilt, gleichzeitig aber auch für die Homophobie und Misogynie in seinen Texten kritisiert wird, zeigt Muñoz auf, dass disidentification ermöglicht, Fanon nicht gänzlich verweigern zu müssen. Durch eine Praxis der disidentification können seine antikolonialen Theorien bewahrt und gleichzeitig seine misogyne und homophobe Haltung abgelehnt werden.

Muñoz betont allerdings, dass er disidentification nicht als eine unpolitische Strategie auf der Suche nach einem Mittelweg oder Kompromiss verstanden haben will. Bezug nehmend auf die berühmte Debatte ${ }^{97}$ zwischen Washington und Du Bois verortet er disidentification vielmehr ganz eindeutig auf der Seite von Du Bois: »Its political agenda is clearly indebted to antiassimilationist thought. ${ }^{98}$ Disidentification ist auch insofern keine Mittelwegsstrategie, als das Risiko, eine Utopie zu formulieren, ein zentraler Bestandteil dieser Praxis ist. ${ }^{99}$ Es ist eine Strategie, die immer auch auf eine andere queere Zukünftigkeit verweist. Dieses Risiko ist auch notwendig, weil disidentification eine Überlebensstrategie ist, die allerdings nicht immer und zwangsläufig für alle sinnvoll beziehungsweise möglich ist: »disidentification is about cultural, material, and psychic survival.« ${ }^{100}$ Am Beispiel eines Projektes des Künstlers* Félix GonzálezTorres zeigt Muñoz auf, inwiefern durch die Praxis der disidentification eine Gegenöffentlichkeit artikuliert werden kann, die nicht nur bestimme identitätspolitische Grup-

J. E. Muñoz: Disidentifications, S. 11.

Ebd., S. 33.

Die Debatte fand zwischen zwei zentralen Figuren der African American Bewegung in den USA zu Beginn des 20. Jahrhunderts statt. Während Washington der Meinung war, Schwarze sollten sich in Bereichen der Wirtschaft und Bildung beweisen, um ihr Recht auf Bürgerrechte einzufordern, forcierte De Bois in der »NAACP« (»National Association for the Advancement of Colored People«) separierende, die Community stärkende Politiken. Während erstere Position als die Verkörperung assimilatorischer Politiken gilt, gelten letztere als die Verkörperung anti-assimilatorischer Politiken.

J. E. Muñoz : Disidentifications, S. 18.

Ebd., S. 25.

Ebd., S. 161. 
pen affiziert. Félix González-Torres hat 1991 an 24 Orten in New York ein »Billboard« veröffentlicht. Auf dem Bild ist ein leeres ungemachtes Bett zu sehen: „two pillows marked with the indention of two absent heads «. ${ }^{101}$ Die Plakate, die nur eine Abwesenheit zeigen, repräsentieren die Verluste der queeren und Latino-Communitys während der Aids-Krise und bringen das Private - das Bett - in die Öffentlichkeit: »Private loss is restructured and becomes public art." Es gebe auf dem Plakat nichts, mit dem mensch sich identifizieren kann, so Muñoz. Es zeige nur eine Abwesenheit, die Gefühle in bestimmten queeren und Latino-, potentiell aber auch in anderen Communitys aufruft: "What is evoked is a structure of feelings that cuts through certain latino and queer communities but is no way exclusive to any identitarian group. " Die Billboards stellen eine Praxis der disidentification dar, mit der dominante Vorstellungen einer Öffentlichkeit/Privatheit-Binarität unterlaufen werden und die jenseits von identitätspolitischer Exklusivität affiziert. ${ }^{102}$ Disidentification ist eine »everyday life«-Praxis, in der durch das gleichzeitige Arbeiten an den, mit den und gegen die Anrufungen, die uns auf einen Platz verweisen, neue Gegenöffentlichkeiten produziert werden können und eine alternative queere Zukunft imaginiert wird.

Politiken des Theatralisierens (Butler) und der disidentification (Muñoz) sind Möglichkeiten, den Platz, auf den Identitäten - oder auch die auf Schließung abzielende Struktur der Anrufung - verweisen, zu unterlaufen und umzuarbeiten. Diese Praktiken der Verkennung, des Umdeutens, des Unterlaufens und Offenlegens können eine alternative, nicht derart um Identitäten angeordnete Zukunft imaginieren, die auch das gemeinsame Band einer kollektiven politischen Gemeinschaft zu knüpfen vermag. Vor allem Muñoz betont, dass disidentification eine Überlebensstrategie sei. Aber auch Butlers Überlegungen zur theatralischen Zitierung des Todes im Kontext der Aids-Krise werden insofern als Überlebensstrategien verstanden, als sie kollektive, fürs Überleben notwendige Trauerprozesse in Gang setzten. Auch Politiken der pluralen Performativität sind unter der Bedingung, dass manche Körper mehr gefährdet sind zu sterben als andere, Kämpfe um ein lebbares Leben aller. Es geht darum, ein lebbares Leben für diejenigen Körper einzufordern, deren Verluste unbetrauerbar sind. Im Zentrum dieser Überlegungen steht weniger der Versuch, durch Umdeutungen/Umarbeitungen eine alternative Zukünftigkeit zu imaginieren, stattdessen steht der Körper in seiner konstitutiven Verletzlichkeit im Zentrum des Politischen.

\subsubsection{Plurale Performativität unter den Bedingungen der Prekarität}

Angesichts aktueller Kriege führt Judith Butler ihre Überlegungen zur Unbetrauerbarkeit von Verlusten jenseits von Psychoanalyse und Geschlechtertheorie fort, indem sie die Frage ins Zentrum rückt, welche Leben als menschlich und betrauerbar gelten und welche nicht. Zentral für diese Auseinandersetzungen ist der in »Raster des Krieges« erstmals verwendete Begriff der Prekarität, der die ungleiche Verteilung von Gefährdetheit, die eng mit der Frage verknüpft ist, welche Leben als lebenswert gelten und

101 Ebd., S. 170.

102 Ebd. 
welche nicht, bezeichnet. ${ }^{103}$ In dem 2015 auf Englisch und 2016 auf Deutsch erschienenen Werk »Anmerkungen zu einer performativen Theorie der Versammlung « führt sie das Konzept der »Prekarität« mit den aus ihren geschlechtertheoretischen Auseinandersetzungen hervorgegangenen Überlegungen zur Performativität zusammen und entwickelt ausgehend von dieser Verknüpfung ein neues Verständnis von politischer Handlungsfähigkeit als "plurale Performativität«.

\section{Der enteignete Körper als Mittel und Ziel des Politischen}

Butler entwickelt ihre Vorstellungen von pluraler Performativität vor dem Hintergrund verschiedener aktueller Protestbewegungen, wie der Proteste am Gezi-Park, TahrirPlatz, Majdan oder der Occupy-Bewegung. Im Zentrum ihrer Überlegungen steht die Frage nach den Voraussetzungen, die gegeben sein müssen, um »auf der Straße zu erscheinen " - also am Politischen teilzuhaben. ${ }^{104}$ Ihr Fokus liegt dabei auf Politiken des Körpers, die Sprechakte vollziehen können, deren Handlungen und Gesten aber auch über das Sprachliche hinausgehen. Bezug nehmend auf Arendts Überlegungen, dass das Politische einen Erscheinungsraum benötige, zeigt Butler auf, dass Körper, die auf der Straße erscheinen, im Erscheinen den Erscheinungsraum des Politischen herstellen. Das gemeinsame Erscheinen von Körpern, das auch visuell oder in nicht öffentlichen Räumen stattfinden kann (beispielsweise, wenn die Risiken, sich zu exponieren, zu groß sind oder es aufgrund eines Gefängnisaufenthalts unmöglich ist, auf die Straße zu gehen), ist bereits insofern performativ, als der politische Erscheinungsraum im Akt der gemeinsamen Versammlung auf der Straße hergestellt wird. ${ }^{105}$ Der Körper wird dabei zugleich als Mittel und Ziel des Politischen verstanden. ${ }^{106}$ Das Erscheinen von Körpern auf der Straße, als Mittel des Protests, setzt bestimmte materielle Bedingungen voraus (z.B. gepflasterte Straßen, Gewaltfreiheit, ökonomische Sicherheit, Bildung, soziale Infrastrukturen und Netzwerke). ${ }^{107}$ Dabei wird der Körper insofern zum Ziel des Protests, als im Erscheinen diese notwendigen Bedingungen eingefordert werden. Das zeigt sich beispielsweise bei den Frauenstreiks, die aktuell in vielen Ländern der Welt stattfinden. Wenn Frauen gemeinsam auf der Straße erscheinen, bleiben viele der als privat geltenden Aufgaben der Sorgearbeit unerledigt. Es kann schwierig sein, auf der Straße zu erscheinen - also den Frauenkörper als Mittel des Protestes einzusetzen -, wenn beispielsweise die Kinderbetreuung oder andere Sorgearbeiten nicht gesichert sind. Gleichzeitig ist der Körper insofern Ziel des politischen Protests, als die Frauenkörper auf der Straße zeigen, dass sie nicht derart »verfügbar sind «. ${ }^{108}$ Indem sie auf der Straße erscheinen und eine Abwesenheit im Privaten markieren, machen

103 Judith Butler: Raster des Krieges. Warum wir nicht jedes Leid beklagen, Frankfurt a.M.: Campus Verlag 2010, S. $31 \mathrm{f}$.

104 Damit ist Butler in diesen Ausführungen den Überlegungen von Spivak nahe, die mit ihrem Begriff der Subalternen diejenigen in den Fokus rückt, die von jeder Möglichkeit zu sprechen, von jeglichen Linien der sozialen Mobilität abgeschnitten sind.

105 J. Butler: Anmerkungen zu einer performativen Theorie der Versammlung, S. 38 u. 105.

106 Ebd., S. 170.

107 Ebd., S. 128.

108 Ebd., S. 38. 
die Körper eine Verweigerung der unbezahlten Sorgearbeit geltend. ${ }^{109}$ Das gemeinsame Erscheinen von Frauenkörpern auf der Straße ist insofern performativ, als sie das Recht einfordern, nicht länger alleine für unbezahlte Care-Arbeiten zuständig zu sein, wenn sie sich genau dieses Recht in dem Moment, in dem sie auf der Straße erscheinen, nehmen. Die politische Forderung wird zugleich inszeniert und gestellt. ${ }^{110}$

Wenn Körper auf diese Art und Weise gemeinsam auf der Straße erscheinen, spricht Butler von einer pluralen Performativität. Der performative Charakter wurde bereits angerissen: Frauenkörper, die streiken, machen das Recht, zum Beispiel nicht derart für Sorgearbeiten zuständig zu sein oder sich frei von (sexueller) Gewalt auf der Straße bewegen zu können, geltend, das sie gleichzeitig einfordern. Das »Plurale« bezieht sich auf das, was »im Dazwischen« - zwischen Körpern - hergestellt wird, nämlich auf den politischen Erscheinungsraum. ${ }^{111}$ Indem Körper sich gemeinsam auf der Straße versammeln, wird der Erscheinungsraum in den Beziehungen, die die Körper miteinander eingehen, hergestellt: »Der >wirkliche< Raum liegt also >zwischen den Menschen, was bedeutet, dass zwar jede Handlung an einem geografisch bestimmbaren Ort stattfindet, darüber hinaus aber auch einen Raum schafft, der eigentlich der Allianz gehört. «112 Obwohl in Butlers Überlegungen der körperliche Akt performativ bestimmte geschlossene Wirklichkeiten herstellt und insofern Mittel des Protestes ist, als er im Zitieren die geschlossene Struktur im Namen einer anderen Zukünftigkeit offenzuhalten versucht, ist nicht die Zukünftigkeit, sondern der Körper selbst Zweck von Butlers aktuellen Überlegungen des Politischen. Es ist auch weniger die Wiederholung, die eine potentielle Öffnung möglich macht, sondern mehr die Offenheit des Körpers selbst, auf der die Möglichkeiten des Widerstandes beruhen.

Butler versteht nicht nur den Erscheinungsraum als etwas, das in Beziehungen, die Körper miteinander eingehen, wenn sie sich auf der Straße versammeln, entsteht, sondern auch den Körper selbst als »lebendige Menge von Beziehungen «. ${ }^{13}$ Körper sind von Anfang an »enteignet «, insofern, als sie nur in Beziehung zu den Anderen ${ }^{114}$ und den sozialen und politischen Infrastrukturen existieren, die ihnen vorgängig sind und die sie nicht gewählt haben. ${ }^{115}$ Dabei handelt es sich um ökonomische und historisch spezifische Beziehungen, die Prekarität und Gewalt, aber auch Leidenschaften und Freundschaften umfassen. ${ }^{116}$ Indem der Körper enteignet und ausgesetzt ist, ist er Butler zufolge immer anfällig für Verwundbarkeiten. Körper sind verwundbar, weil

109 Sie zeigen auch, dass ihr Körper nicht für ungleiche Löhne oder (sexuelle) Gewalt verfügbar sind.

110 J. Butler: Anmerkungen zu einer performativen Theorie der Versammlung, S. 181.

111 G. Posselt: Politiken des Performativen.

112 J. Butler: Anmerkungen zu einer performativen Theorie der Versammlung, S. 99.

113 Ebd., S. 88.

114 Körper werden bei Butler allerdings nicht als eine abgeschlossene Kategorie des Menschlichen verstanden. Insofern der Körper konstitutiv in und durch Beziehungen zu seiner Umwelt, der Technik, den sozialen Infrastrukturen hergestellt wird, ist er außer sich und befindet sich in einer wechselseitigen Abhängigkeit zu dem, was als nichtmenschlich gilt. Körper sind in gewisser Weise also auch abhängig von einer Infrastruktur, »dem komplexen Geflecht aus Umwelt, Sozialbeziehungen, Unterstützungs- und Versorgungsnetzwerken.« Ebd., S. 175.

115 Ebd., S. 130

116 Ebd., S. 194. 
sie offen sind für das Geschehen und damit auch für das, was unvorhersehbar geschehen kann. Es sind keine "geschlossenen Entitäten«, sondern sie sind offen gegenüber anderen und der Welt: »Unsere Empfänglichkeit für alles, was geschieht, ist eine Funktion und eine Wirkung der Vulnerabilität - des Offenseins gegenüber der Geschichte, des Registrierens eines Eindrucks oder des Beeindrucktwerdens. "117 Während Judith Butler die aus unserer Verwundbarkeit resultierende Verletzlichkeit als eine universelle Kategorie versteht, ist Prekarität ein Begriff, der die Gefährdetheit beschreibt, die durch politische und gesellschaftliche Verhältnisse strukturiert ist. ${ }^{118}$

\section{Prekarität und Performativität}

Prekarität beschreibt »den politisch bedingten Zustand, in dem bestimmte Teile der Bevölkerung unter dem Versagen sozialer und ökonomischer Unterstützungsnetze mehr leiden und anders von Verletzung, Gewalt und Tod betroffen sind als andere ${ }^{119} \mathrm{Da}$ bei ist Prekarität nicht nur materiell und ökonomisch, sondern auch eine affektive Gefühlslage der »Entbehrlichkeit« und »Verfügbarkeit« - ein Gefühl der Angst, nicht für sich selbst oder die, die von einem abhängig sind, sorgen zu können. ${ }^{120}$ Prekarität beschreibt deshalb keine Identität, sondern vielmehr einen gemeinsamen Zustand, der Ausgangspunkt für gemeinsames Handeln jenseits identitätspolitischer Festschreibungen ist. ${ }^{121}$ Unter den Bedingungen der Prekarität kann die Verletzlichkeit von Körpern gleichzeitig Ziel und Mittel des Politischen sein. ${ }^{122}$ Auf der Straße zu erscheinen, bedeutet oft, den eigenen Körper der Gefahr auszusetzen. ${ }^{123}$ Illegale Arbeiter*innen, Staatenlose, Frauen oder Queers legen die Verletzlichkeit ihrer Körper offen, wenn sie gemeinsam auf der Straße erscheinen. An diesem Punkt verbindet sich das Moment der Prekarität mit dem der Performativität. Indem die Verletzlichkeit offengelegt wird, wird performativ das Recht eingefordert, ohne Gefahr auf der Straße zu erscheinen. Unter den Bedingungen der Prekarität das Recht zu erscheinen in Anspruch zu nehmen, kann bedeuten, gemeinsam für ein gleiches Leben, ein lebbares Leben für alle auf der Straße zu erscheinen. Es bedeutet für die Möglichkeitsbedingungen des Erscheinens selbst, in Erscheinung zu treten: »Ohne diese Unterstützung in ihrem Namen zu handeln ist das Paradox des pluralen performativen Handelns unter den Bedingungen der Prekarität. « ${ }^{124}$ Es wird der Widerspruch eines demokratischen öffentlichen Raums, der über Ausschlüsse konstituiert ist, offengelegt, indem die, die nicht das Recht haben $\mathrm{zu}$ erscheinen, im Erscheinen ihr Recht zu erscheinen einfordern. Es geht nicht

117 Ebd., S. 195.

118 J. Butler: Raster des Krieges, S. $31 \mathrm{f}$.

119 J. Butler: Anmerkungen zu einer performativen Theorie der Versammlung, S. 40.

120 Ebd., S. 24.

121 Ebd., S. 40.

122 Prekarität ins Zentrum des Politischen zu stellen, markiert allerdings keinen Bruch zu Butlers früheren Überlegungen zu Geschlechterkämpfen. Im Gegenteil: Prekarität ist auch insofern mit Geschlecht verknüpft, als manche vergeschlechtlichte Körper mehr Gefahr ausgesetzt sind, wenn sie im öffentlichen Raum erscheinen, als andere. Das bedeutet, Geschlecht und Sexualität kann ein Merkmal sein, über das Gefährdung strukturiert ist. Ebd., S. 49. 
so sehr darum, einen Platz für die Ausgeschlossenen einzufordern, sondern die Lücke offenzulegen, die den »Universalitätsanspruch entkräftet«. ${ }^{125}$

\section{Queere "Koalitionen des Überlebens « ${ }^{126}$}

Wenn gefährdete Körper gemeinsam auf der Straße erscheinen, wird aber nicht nur die Verletzlichkeit des Körpers offengelegt, sondern auch die Gemeinschaftlichkeit das Abhängige der Körper - inszeniert. Die Möglichkeit, auf Grundlage einer konstitutiven Abhängigkeit voneinander Allianzen jenseits von Identitäten zu schmieden, stellt eine weitere Ebene dar, auf der die Verletzlichkeit des Körpers in Butlers Denken des Politischen zentral ist. Im Namen und unter den Bedingungen von Prekarität zu handeln, bedeutet nicht, aus einer Autonomie, sondern aus einer Abhängigkeit ${ }^{127}$ heraus $\mathrm{zu}$ agieren. ${ }^{128}$ Prekarität ist dabei ein Begriff, der gleichzeitig trennt, indem er Ungleichheiten markiert, und verbindet, insofern er von einer wechselseitigen Abhängigkeit ausgeht. ${ }^{129}$ Während Butler in den bisher angeführten geschlechtertheoretischen Arbeiten die Verleugnung der leidenschaftlichen Verhaftung an die anderen vor allem als einen Moment beschreibt, der im Prozess der Subjektwerdung verdrängt und verleugnet wird und damit gleichzeitig das Psychische formiert, steht in ihren aktuellsten Überlegungen ebendiese Abhängigkeit im Zentrum des gemeinsamen Bandes, das zwischen Allianzen geknüpft wird: »Solidarität in der Demokratie ist daher nur zu haben um den Preis der Enteignung - gerade weil wir immer schon Enteignete sind. « ${ }^{130}$

»Prekarität als Stoff für queere Koalitionen« bietet die Möglichkeit, ein kollektives Wir jenseits der »liberalen Sackgasse« herzustellen. Es geht darum, den durch neoliberale Ideologien hervorgebrachten Gefühlen der »Angst und des Scheiterns« eine gemeinsame Verantwortlichkeit füreinander entgegenzusetzen. Indem in Allianzen die Enteignung zum Ausgangspunkt politischer Handlungsfähigkeit gemacht wird, kann eine Sozialität hergestellt werden, in der liberale Grundwerte von Autonomie und Eigentum anders denkbar sind. Es wird möglich, einen Autonomiebegriff in Frage zu stellen, der vor allem ein »Privileg der Besitzenden« ist. Solidarische Bündnispolitiken stellen das für das Kapital grundlegende Verhältnis von Autonomie und Eigentum in Frage und schaffen eine Gegenerzählung zu liberalen Vorstellungen von Autonomie und Eigenverantwortlichkeit. Damit wird ein liberales Verfahren, in dem Individuen zu

125 Ebd., S. 70.

126 Sabine Hark: Koalitionen des Überlebens. Queere Bündnispolitiken im 21. Jahrhundert, Göttingen: Wallstein Verlag 2017, S. 22.

127 Abhängigkeit ist für Butler also nicht das, was unserer Freiheit entgegensteht. Im Cegenteil, gerade dadurch, dass wir voneinander abhängig sind - in einer Sozialität eingebunden sind -, können wir auf Unterstützungs- und Versorgungsnetzwerke zurückgreifen, die unsere Freiheit überhaupt erst ermöglichen, beziehungsweise haben wir so die Freiheit, diese Infrastrukturen zu fordern. Das Problem ist nicht unsere Abhängigkeit an sich, sondern die taktische Ausnutzung von Abhängigkeit, die Ausbeutung von Abhängigkeit. ]. Butler: Anmerkungen zu einer performativen Theorie der Versammlung, S. 193.

128 Ebd., S. 63.

129 S. Hark: Koalitionen des Überlebens, S. 22.

130 Ebd., S. 53. 
Subjekten werden, zur Disposition gebracht. ${ }^{131}$ Gleichzeitig sind diese Bündnisse insofern performativ, als sie eine andere noch nicht festgeschriebene Sozialität herstellen, die im Akt des beständigen Erschaffens eingefordert wird. ${ }^{132}$ Es gilt also, Bündnisse zu schließen, die auf einer gemeinsamen Enteignung beruhen, in denen eine neue Sozialität hergestellt und imaginierbar wird.

Doch auch das trennende Moment von Prekarität schreibt sich in Koalitionen ein. Jinthana Haritaworn warnt beispielsweise vor der Gefahr, dass Allianzen mehrfachminorisierte Subjekte abermals in den Schatten stellen, wenn Machtverhältnisse unhinterfragt bleiben:»Die zumeist an widerspenstige Minorisierte herangetragene Forderung, sich mit Majorisierten jeglicher politischen Positionierung zu verbünden, leugnet den Schmerz, das Risiko und die Gefahr, die damit verbunden ist, sich auf seine UnterdrückerInnen zu bewegen [sic!], nur um wieder zurückgestoßen, bevormundet oder dämonisiert zu werden. ${ }^{133}$ Koalitionen sind - das betont auch Judith Butler - gerade, weil wir mit denen zusammenarbeiten, die wir nicht gewählt haben, harte Arbeit, die von Gefühlen der Bedrohung und Angst begleitet sein kann. Die afroamerikanische Feministin Johnson Reagon zitierend schreibt sie:

»Meistens fühlt man sich zutiefst bedroht, und wenn man das nicht fühlt, dann ist es keine echte Koalitionsarbeit. [...] Man geht keine Koalition ein, nur, weil es einem einfach gefällt. Der einzige Grund, eine Zusammenarbeit mit jemanden, der dich womöglich töten kann, überhaupt in Erwähnung zu ziehen, ist, dass du dir keine andere Möglichkeit vorstellen kannst, am Leben zu bleiben. « ${ }^{134}$

Koalitionen im Namen von Prekarität bedeuten also nicht, dass alle gleich sein oder die gleichen Interessen haben müssen. Doch so sehr es auch darum geht, Differenzen in koalitionären Politiken nicht auszulöschen, müssen auch die Punkte, an denen die verschiedenen Geschichten der Unterdrückung miteinander verwoben sind, betont werden: »Was wir uns daher, mit anderen Worten, nicht erlauben können, ist, weder radikal verschiedene Geschichten unser eigen [sic!] zu nennen, noch uns als in absolut heterogenen und getrennten Orten lebend $\mathrm{zu}$ imaginieren. ${ }^{135}$

Ein Punkt, an dem die verschiedenen Geschichten konstitutiv miteinander verwoben sind, ist, dass Menschen gemeinsam mit denen, die sie nicht gewählt haben, die Erde bevölkern. Wenn - hier bezieht sich Butler erneut auf Hannah Arendt - die irdischen Bedingungen des Seins zwar ungewählt, aber doch die Bedingungen für Pluralität und das Zusammenleben sind, dann sind Menschen darauf angewiesen, mit denen zusammenzuarbeiten, die ihnen nicht nahe sind und die sie nicht gewählt haben. ${ }^{136}$ Nicht aus Liebe oder Menschlichkeit, sondern weil sie insofern voneinander abhängig sind, als die politischen Bedingungen des Seins darauf beruhen, dass Menschen gemeinsam

Ebd., S. 47.

Ebd., S. 48.

]. Haritaworn: Am Anfang war Audre Lorde. Weißsein und Machtvermeidung in der queeren Ursprungsgeschichte* ${ }^{*}$ S. 32 .

J. Butler: Anmerkungen zu einer performativen Theorie der Versammlung, S. 198

S. Hark: Koalitionen des Überlebens, S. 55.

J. Butler: Anmerkungen zu einer performativen Theorie der Versammlung, S. 148 
die Erde bevölkern. Auch Emmanuel Levinas, auf den Butler sich bezieht, spricht davon, dass Menschen insofern, als sie bereits vor jeder Anrufung aneinandergebunden sind, füreinander verantwortlich sind. Das Ich, das in der Anrufung hergestellt wird, steht, Levinas zufolge, von Anfang an in einer Beziehung mit dem Du: $D$ Du rufst mich an und ich antworte. Wenn ich jedoch antworte, dann nur, weil ich schon verantwortlich war; das heißt diese Empfänglichkeit und Verwundbarkeit konstituiert mich auf der fundamentalsten Ebene und besteht, so könnte man sagen, schon vor jeder bewussten Entscheidung, auf den Ruf zu antworten. " 137 Ausgehend von diesen Überlegungen von Levinas und Arendt verknüpft Judith Butler das demokratische Prinzip der Offenheit mit einer Ethik. Die Bindung an den anderen verweist Levinas folgend auf eine ethische Verantwortung gegenüber den anderen, unabhängig davon, wie nah wir ihnen sind. Es geht darum, gemeinsam für ein lebbares Leben für alle in ihrer Unterschiedlichkeit zu kämpfen, weil wir immer schon und von Anfang an selbst auch gebunden und abhängig sind von denen, deren Leben (Umwelt) von den Bedingungen der Prekarität gefährdet sind. Gegen eine »unternehmerische Ethik«, nach der die Machtlosen für sich selbst verantwortlich sind, sieht Butler in Versammlungen von Körpern auf der Straße einen »Grund zum Jubeln«, wenn sie die Einsicht teilen, dass »Prekarität differentiell verteilt ist « und der Widerstand gegen Prekarität auf dem Anspruch basiert, dass alle »Leben gleich behandelt werden und gleich lebbar sein sollen «. ${ }^{138}$ Allianzen können trotz Unterschiedlichkeiten und Distanzen, auf Basis einer ethischen Verantwortung füreinander, aneinandergebunden werden. Dabei sollten solche Allianzen und Gemeinschaften selbst die Werte verkörpern, die sie einfordern. ${ }^{139}$

Butler entwickelt in ihrem jüngsten Werk, ausgehend von aktuellen Protestbewegungen, eine Theorie des Politischen, indem sie ihre Überlegungen zur Performativität wieder aufgreift und mit dem Konzept Prekarität in Beziehung setzt. Dabei zeigt sie, häufig auch Bespiele von queeren Kämpfen verwendend, dass Politiken im Namen von Prekarität im Grunde »queer« sind. Es ist weniger die »Queerness«, die sich in Butlers früheren Werken nicht »allzu sehr von den Schlenkern der Iterabilität [...] unterscheidet «, ${ }^{140}$ sondern vielmehr die Verwundbarkeit als Resultat unserer Offenheit - die wiederum auch als »Queerness« bezeichnet werden kann -, die politische Handlungsfähigkeit möglich macht. Die hier vorgetragenen Theoretisierungen des Widerstandes bedienen sich immer wieder des Beispiels queerer Aids-Politiken. Einer queeren Bewegungsgeschichte zufolge waren Allianzen angesichts unbetrauerbarer Verluste in der Aids-Krise von Anfang an ein zentraler Aspekt queeren Widerstandes. ${ }^{141}$ Nachdem nun die »theoretische« Seite beleuchtet wurde, soll es im Folgenden darum gehen, einen Blick auf die queere Bewegungsgeschichte zu werfen, durch die nicht nur queere Theoriebildung, sondern auch aktuelle queere Politiken geworden sind, was sie sind.

137 Ebd., S. 147

138 Ebd., S. 92.

139 Ebd., S. 93.

140 Ebd., S. 85.

141 Bereits in »Gender Trouble«verweist Judith Butler auf Bündnispolitiken als eine mögliche Antwort auf identitätspolitische Dilemmata. Vgl. J. Butler: Das Unbehagen der Geschlechter, S. 34. 